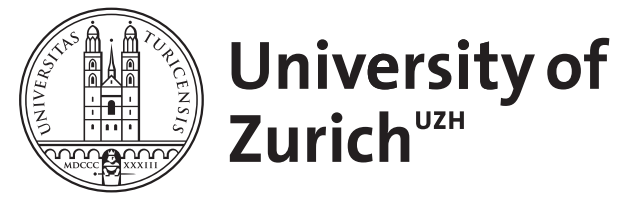

\title{
Fragen an einen Weggefährten
}

Fischer, Johannes

DOI: https://doi.org/10.1515/9783110247893.343

Posted at the Zurich Open Repository and Archive, University of Zurich

ZORA URL: https://doi.org/10.5167/uzh-50766

Book Section

Published Version

Originally published at:

Fischer, Johannes (2011). Fragen an einen Weggefährten. In: Polke, Christian; Brunn, Frank Martin; Dietz, Alexander; Rolf, Sibylle; Siebert, Anja. Niemand ist eine Insel. Berlin / Bosten: Walter de Gruyter GmbH, 343-358.

DOI: https://doi.org/10.1515/9783110247893.343 


\title{
Fragen an einen Weggefährten
}

\author{
Johannes Fischer
}

Manchmal tritt der denkwürdige Fall ein, dass aus Trennendem Verbindendes hervorgeht und dass sich aus Kontroversen Weggefährtenschaft entwickelt. Das ist zum Beispiel der Fall, wenn zwei Menschen sich, ohne ihre Differenzen überwinden und ausräumen zu können, aufgrund besonderer Umstände dennoch zusammenfinden und auf etwas verständigen müssen, dem sie aus innerer Überzeugung gemeinsam zustimmen können. Von dieser Art ist die Verbundenheit, die ich in Bezug auf Wilfried Härle empfinde, und das Wort, Weggefährtenschaft' gibt dieser Verbundenheit treffend Ausdruck. In den Jahren, in denen Wilfried Härle sich als Vorsitzender der Kammer für öffentliche Verantwortung der Evangelischen Kirche in Deutschland (EKD) große Verdienste erworben hat, haben wir so manche Kontroverse ausgefochten. Es ging um Stammzellforschung, Präimplantationsdiagnostik oder Sterbehilfe, um Menschenwürde oder das evangelische Verständnis der Person, und im Hintergrund standen unsere unterschiedlichen Auffassungen von Theologie und Ethik. Bei der Abfassung der bio- und medizinethischen Texte der Kammer haben wir bis zuletzt um einzelne Formulierungen gerungen. Gemeinsam war uns die Überzeugung, dass unsere Differenzen nicht zur Blockade für die Arbeit der Kammer werden durften, sondern dass wir konstruktive Lösungen finden mussten. Vielleicht brachte sich in diesem Bemühen etwas davon zur Geltung, dass der Geist, aus dem die evangelische Kirche lebt, sich nicht nur in ihren Konsensen zeigt, sondern mehr noch in der Art und Weise, wie sie mit Dissens umzugehen versteht, nämlich in der Haltung des Respekts vor dem, der anders denkt. So gesehen fällt gerade vom Trennenden her ein umso helleres Licht auf das Verbindende, dem das Trennende, so mühsam, ja belastend es auch manchmal gewesen ist, doch nichts anhaben kann.

Gemeinsam ist uns die Überzeugung, dass Gegensätze nicht übertüncht oder unter dem Teppich gekehrt werden dürfen. Ich habe über die Jahre die Beharrlichkeit schätzen gelernt, mit der Wilfried Härle nach- 
fragte und verstehen wollte, was genau es ist, das mich anderer Meinung sein ließ als er, und ob es sich dabei tatsächlich um unüberbrückbare Gegensätze handelte. Das zwang dazu, die eigenen Positionen zu überdenken, und es hat der Klärung gedient. Es entspricht diesem spezifischen Charakter meiner Verbundenheit mit Wilfried Härle, wenn ich ihr im Folgenden in der Weise Ausdruck gebe, dass ich aus meiner Sicht so klar und prägnant, wie es mir möglich ist, zu benennen versuche, was uns in unseren Auffassungen von Theologie und Ethik trotz aller Verständigungsbemühungen trennt. Ich betrachte dies als Teil der kritischen Weggefährtenschaft, die mich mit ihm verbindet.

Würde man Wilfried Härle und mich auffordern, den Grundzug des Denkens des jeweils anderen zu benennen, an dem sich die eigene Kritik entzündet, dann würde er mich vermutlich als einen „Subjektivisten“ charakterisieren - sicherlich ohne diesen Ausdruck zu verwenden, dazu ist er Schlagworten gegenüber viel zu reserviert und sprachgenau, aber doch der Sache nach -, der dasjenige, worum es auf dem Gebiet des Glaubens und der Moral geht, in der Wahrnehmung, Intuition oder dem Gefühl fundiert, während ich ihn als einen „Objektivisten“ charakterisieren würde, der die Wahrheit des Glaubens und der Moral in Bedingungen aufsucht, die all unserem subjektiven Wahrnehmen, Erkennen oder Fühlen verbindlich vorgegeben sind. Vermutlich würden wir uns dann beide gegen diese Charakterisierungen entschieden verwahren, da wir unser eigenes Denken als sehr viel differenzierter begreifen. Aber ich glaube doch, dass sie etwas treffen von dem, was uns in unserem theologischen und ethischen Denken trennt und was im Hintergrund unserer Kontroversen gestanden ist.

Ich kann nur für meine Sicht sprechen, und ich will diese zunächst an Wilfried Härles Konzeption der Theologie verdeutlichen. Wilfried Härle begreift die Theologie als eine Wirklichkeitswissenschaft. ${ }^{1}$ Als solche hat

1 Ich verwende diesen Ausdruck in dem Sinne, wie ihn Heinrich ScHolz gebraucht hat, vgl. Ders., Wie ist evangelische Theologie als Wissenschaft möglich?, in: Zwischen den Zeiten 9 (1931), 8-53. Wirklichkeitswissenschaften sind z. B. die Physik oder die Soziologie im Unterschied zur Mathematik oder Logik. Auch Scholz war bei seinen Anfragen an die Theologie Karl Barths der Meinung, dass die evangelische Theologie, wenn sie denn eine Wissenschaft ist, eine 
sie es mit Gott und der durch Gott bestimmten Wirklichkeit zu tun. Sie teilt dabei die Perspektive des Glaubens beziehungsweise ,geschieht aus der Perspektive des Glaubens heraus"2. Als Wissenschaft macht sie sich die Überprüfung der Wahrheit der Glaubensaussagen zur Aufgabe. Methodisch verfährt sie dabei nach dem deduktiv-hypothetischen Wissenschaftsmodell, wie es durch Karl Popper formuliert worden ist. Gemäss diesem Modell hat sie die Aussagen des christlichen Glaubens als „Hypothesen“"3 zu „bewähren“, indem sie sie mitsamt ihren logischen Implikationen der Möglichkeit der Falsifizierung aussetzt. ${ }^{4}$

Diese Auffassung von Theologie wirft aus meiner Sicht eine Reihe von Fragen auf. Erstens: Wie kann die wissenschaftliche Theologie einerseits die Perspektive des christlichen Glaubens teilen und andererseits nach dem deduktiv-hypothetischen Wissenschaftsmodell verfahren? Der Glaube unterstellt die Wirklichkeit dessen, woran er glaubt, mit kategorischer Bestimmtheit. Wissenschaftliche Hypothesen sind demgegenüber keine kategorischen, sondern hypothetische Wahrheitsunterstellungen: ,Angenommen, A ist der Fall: Welche Konsequenzen B, C, D usw. ergeben sich hieraus?‘. Ein Wissenschaftler, der von der Wahrheit einer bestimmten Aussage ausgeht, behandelt diese gerade nicht als eine Hypothese. Was soll daher gelten: Dass die wissenschaftliche Theologie aus der Perspektive des Glaubens heraus geschieht, also diese Perspektive teilt? Oder dass sie nach dem deduktiv-hypothetischen Modell verfährt und dementsprechend gerade nicht von der Wahrheit der Glaubensaussagen ausgeht, sondern diese als Hypothesen behandelt?

Damit steht ein zweites Problem in engem Zusammenhang. Wenn die wissenschaftliche Theologie die Perspektive des Glaubens teilt, dann ist ihr Gegenstand Gott beziehungsweise die durch Gott bestimmte Wirklichkeit. Verfährt sie hingegen nach dem deduktiv-hypothetischen Modell, dann ist ihr Gegenstand der Gottesgedanke beziehungsweise der Gedanke der durch Gott bestimmten Wirklichkeit, den sie zu bewähren sucht, indem sie seine logischen Implikationen dem Falsifizierungstest unterwirft. ${ }^{5}$ Was also ist Gegenstand der wissenschaftlichen Theologie: Gott oder der Gottesgedanke?

Wirklichkeitswissenschaft ist. Vgl. zu dieser Auffassung auch Wolfhart PANNENBERG, Wissenschaftstheorie und Theologie, Frankfurt a.M. 1977.

2 Wilfried Härle, Dogmatik, Berlin/ New York 1995, 10.

3 A.a.O., $22 \mathrm{f}$.

4 Vgl. a.a.O., $23 \mathrm{f}$.

5 Vgl. Pannenberg, Wissenschaftstheorie und Theologie (s. o. Anm. 1), $301 \mathrm{f}$. 
Dies führt zu einem dritten Problem: Um als Hypothesen aufgefasst werden zu können, müssten Glaubensaussagen Urteilscharakter haben. Urteile sind durch einen selbstbezüglichen Anspruch hinsichtlich der Wahrheit der Aussage charakterisiert, die sie formulieren. Mit dem Urteil ,Angela Merkel ist die Kanzlerin der Bundesrepublik Deutschland“ wird der Anspruch erhoben, dass die Aussage ,Angela Merkel ist die Kanzlerin der Bundesrepublik Deutschland 'wahr ist. Sind Glaubensaussagen von dieser Art? Wilfried Härles Beispiele sind die Aussagen „Herr ist Jesus (Christus)“ (Röm 10, 9 u. ö.), „Er (Jesus Christus) ist auferstanden“ (Mt 28, 6 u. ö.) oder „Du bist Christus, des lebendigen Gottes Sohn!“ (Mt 16, 16), die er wissenschaftstheoretisch als Hypothesen interpretiert. ${ }^{6}$ Ist dies sachgemäss? Falls mit dem gottesdienstlichen Ausruf „Herr ist Jesus (Christus)!“ überhaupt ein Anspruch verbunden ist, dann bezieht sich dieser ersichtlich darauf, dass Jesus Herr ist, nicht aber darauf, dass die Aussage ,Jesus ist Herr' wahr ist. Es wird also mit dieser Glaubensaussage kein Urteil formuliert. ${ }^{7}$ Dasselbe gilt für die anderen beiden Beispiele oder auch für eine Glaubensaussage wie „Denn Gott war in Christus und versöhnte die Welt mit sich selbst“ (II Kor 5, 20). Hierin unterscheiden sich Glaubensaussagen von wissenschaftlichen Feststellungen. Letztere haben Urteilscharakter, und Hypothesen sind Urteile, für die Wahrheit hypothetisch unterstellt wird. Wenn aber Glaubensaussagen gar keinen selbstreflexiven Anspruch auf Wahrheit erheben, dann verlangen sie auch nicht nach irgendeiner Art von Verifikation oder wissenschaftlicher „Bewährung“. Mit dem hypothetisch-deduktiven Modell wird daher etwas an sie herangetragen, was ihrem Wesen zuwider läuft.

Wilfried Härle wäre nicht der sprachsensible Theologe, der er ist, wenn ihm das hier liegende Problem entgangen wäre. „Vom Selbstverständnis des christlichen Glaubens her ist es jedoch missverständlich, ja in gewisser Hinsicht sogar inakzeptabel, solche Fundamentalaussagen als

6 Vgl. Härle, Dogmatik (s. o. Anm. 2), 23.

7 Vielleicht wird man einwenden, dass dies in Bezug auf die Glaubensaussage als solche zwar richtig ist, dass aber gleichwohl derjenige, der eine solche Glaubensaussage macht, implizit ein solches Urteil fällt und sich damit in eine Rechtfertigungspflicht in Bezug auf dessen Wahrheit bringt. Ich halte diese Auffassung für falsch und habe meine diesbezüglichen Argumente entwickelt in: Johannes Fischer, Behaupten oder Bezeugen? Zum Modus des Wahrheitsanspruchs christlicher Rede von Gott, in: ZThK 87 (1990), 224-244. Ich würde diesen Aufsatz heute in mancher Hinsicht anders schreiben und insbesondere nicht mehr von einem „Wahrheitsanspruch christlicher Rede von Gott“ sprechen. Aber die zentrale These scheint mir nach wie vor gültig zu sein. 
,Hypothesen` zu bezeichnen. Für Menschen, die von der Wahrheit und Bedeutung dieser Aussagen ergriffen worden sind, also in existentieller Hinsicht, handelt es sich dabei gerade nicht um Hypothesen (im Sinne von Vermutungen oder nur bedingt gültigen Sätzen), sondern um tragende Gewissheiten, für die nicht wenige Menschen Hab und Gut, ja Leib und Leben aufs Spiel gesetzt und verloren haben. Wenn solche Fundamentalaussagen als ,Hypothesen“ bezeichnet werden, so gilt das ausschliesslich in wissenschaftstheoretischer Hinsicht. Und in dieser Hinsicht ist die Bezeichnung akzeptabel. “8 Wenn freilich der Glaubende dessen existentiell gewiss ist, was jene Aussagen zum Ausdruck bringen, wozu bedarf es dann überhaupt der wissenschaftstheoretischen Transformation solcher Aussagen in hypothetische Urteile, deren Wahrheitsanspruch durch die wissenschaftliche Theologie im Falsifizierungsverfahren ,bewährt“" werden soll?

Im Kern geht es hier um die Frage, wozu es überhaupt wissenschaftliche Theologie braucht. In Bezug auf eine Auffassung von Theologie, welche die wissenschaftliche Überprüfung und Bewährung der Wahrheit des christlichen Glaubens als deren Aufgabe statuiert, haben aus meiner Sicht die Anfragen nach wie vor Gültigkeit, die Franz Overbeck an die Theologie seiner Zeit richtete: „Daher ist denn auch das Thun jeder Theologie, sofern sie den Glauben mit dem Wissen in Berührung bringt, an sich selbst und seiner Zusammensetzung nach ein irreligiöses, und kann keine Theologie jemals entstehen, wo nicht neben das religiöse Interesse sich diesem fremde stellen. ${ }^{\text {"9 }}$ Nach Overbecks Sicht verhalten sich diese fremden Interessen zum Glauben und zur Religion, denen sie zu dienen vorgeben, in Wahrheit rein destruktiv. Dass „das Christenthum für die Theologie ein wissenschaftliches Problem“" ist, bedeutet nach dieser Sicht nichts anderes, ,als dass die Theologie das Christenthum als Religion problematisch macht, das heißt als solche überhaupt in Frage stellt. Und zwar gilt dies von aller Theologie, welches auch ihre Resultate sein mögen. Denn selbst das Resultat, die Ehrlichkeit seiner Gewinnung vorausgesetzt, würde hier die apologetische Theologie mindestens nicht günstiger stellen als die kritische, da auch die apologetische Theologie, wenn von ihr das Christenthum wissenschaftlich bewiesen wäre, es als Religion vernichtet hätte. “10

\footnotetext{
8 Härle, Dogmatik (s. o. Anm. 2), passim.

9 Franz Overbeck, Über die Christlichkeit unserer heutigen Theologie, (Leipzig 1903) Darmstadt ${ }^{3} 1963,24 \mathrm{f}$.

10 A.a.O., 35.
} 
Man muss Overbecks radikal theologiekritische Auffassung nicht teilen, um doch von ihr lernen zu können, dass es sehr gute Gründe braucht, um überhaupt so etwas wie wissenschaftliche Theologie verantworten zu können. Sollen hier nicht ,,neben das religiöse Interesse sich diesem fremde stellen“, dann können diese Gründe nur im christlichen Glauben selbst liegen. Nach dem Gesagten braucht es wissenschaftliche Theologie nicht deshalb, weil der Glaube danach verlangen würde, dass die Wahrheit seiner Aussagen verifiziert oder bewährt wird. Vielmehr gibt es nur einen einzigen im Glauben selbst liegenden Grund, warum es solche Theologie braucht, und das ist die Tatsache, dass das Verständnis von Begriffen, Vorstellungskomplexen, Aussagen oder Gedankenzusammenhängen der Glaubensüberlieferung dunkel und unklar sein kann. Die Klärung dieses Verständnisses - zum Beispiel des Ausdrucks ,Gerechtigkeit Gottes' bei Paulus - ist keine Frage von Glaubensüberzeugungen, sondern etwas, das mit Notwendigkeit auf die Ebene des philologischen, historischen und systematischen Wissens führt und nur auf dieser Ebene erfolgen kann. Wird sie in dieser Weise begriffen, dann ist die wissenschaftliche Theologie keine Wirklichkeitswissenschaft, die Gott zu ihrem Gegenstand hat, sondern eine hermeneutische Disziplin, die das Verständnis Gottes zum Gegenstand hat, wie es in den christlichen Glaubenszeugnissen begegnet. Ihr Ziel ist der gelebte Glaube, den sie zu einem besseren Verstehen dessen anzuleiten sucht, was er glaubt, und zwar nicht bloss im Sinne eines philologischen oder historischen Verstehens, sondern eines existentiellen, das je eigene Leben betreffenden Verstehens. Gott ist hiernach allein Gegenstand solchen Verstehens, das heißt Glaubens, nicht aber des wissenschaftlichen Wissens.

Ich möchte es vorsichtig als Frage formulieren, ob nicht Wilfried Härles Konzeption von Theologie letztlich eine apologetische Zielsetzung zugrunde liegt, nämlich gegenüber denen, die im Rahmen des wissenschaftlichen Weltbilds die Wahrheit des Glaubens bezweifeln und dabei Glaubensaussagen im Sinne von Urteilen missverstehen -, dessen Wahrheit zu erweisen oder zu bewähren. Sollte es sich in dieser Weise verhalten: Muss man sich dann nicht darüber Rechenschaft geben, dass dieses Weltbild massgeblich durch den Siegeszug der Naturwissenschaften geprägt worden ist und dass auch das deduktiv-hypothetische Modell für diese Art von Wissenschaften entwickelt worden ist? Was tut man, wenn man dieses Modell auf die Theologie überträgt? Soll die Theologie ernstlich dem fundamentalen Missverständnis nachgeben, dem sich die Religion im Rahmen dieses Weltbilds ausgesetzt sieht, indem sie die Wahrheit des christlichen Glaubens innerhalb dieses Rahmens wis- 
senschaftlich zu bewähren sucht? Müsste sie nicht vielmehr dieses Missverständnis aufklären, und zwar indem sie die Eigenart von Äusserungen beziehungsweise Aussagen des Glaubens hermeneutisch reflektiert und analysiert und deren fundamentale Unterschiedenheit von Urteilen und insbesondere wissenschaftlichen Urteilen verdeutlicht? Wilfried Härles theologische Konzeption führt jedenfalls zur Einnahme eines anderen Standpunkts jenseits des Glaubens, eben des wissenschaftlichen, von dem aus die Bewährung der Wahrheit der Glaubensaussagen methodisch geleistet werden können soll. Hat Franz Overbeck nicht schärfer gesehen, wenn er feststellt, dass dies in der Konsequenz zur Destruktion von Glaube und Religion führt? Die reformatorische Theologie unterschied zwischen dem credere Deum esse, dem credere Deo und dem credere in Deum (fiducia). Vorausgesetzt, es kann mit dem deduktiv-hypothetischen Modell in theologischer Hinsicht überhaupt etwas gezeigt werden: Kann damit mehr gezeigt werden als das Deum esse in dem weiten Sinne, in dem es Gottes Sein und (zum Beispiel schöpferisches) Handeln als facta mitumfasst? Und soll tatsächlich hierauf der methodische Fokus der wissenschaftlichen Theologie liegen? Führt dies nicht gerade weg von jenem existentiellen credere in Deum, das nach reformatorischer Sicht allein selig macht? Oder sollte ernstlich dies die Meinung sein, dass das credere in Deum die wissenschaftliche Bewährung des Deum esse zur Voraussetzung hat? Wie kann es dies, wenn es selbst gar keinen Urteilscharakter hat? Wenn zwischen beidem aber kein Voraussetzungsverhältnis besteht, wozu braucht es dann diese Art von theologischer Wirklichkeitswissenschaft?

In seiner Dogmatik hat Wilfried Härle freilich seine an Popper geschulte wissenschaftstheoretische Auffassung von Theologie nicht wirklich streng und bis in alle Konsequenzen durchgefuihrt, und es findet sich darin vieles, was ich eher einer hermeneutischen Konzeption von Theologie zuordnen würde. Gerne füge ich hinzu, dass ich daraus manches dankbar gelernt habe. Trotz des Trennenden im Grundsätzlichen unseres Denkens scheint mir eine Verständigung in theologischen Einzelfragen keineswegs unmöglich zu sein. Ich möchte daher die Vermutung wagen, dass wir uns in dem, was uns theologisch antreibt, vielleicht näher stehen, als es unsere fundamentaltheologischen Differenzen erwarten lassen. 
Die Suche nach einem „objektiven“ Standpunkt, der über das persönliche, ,subjektive“ Involviertsein hinausführt und der es erlaubt, unsere Orientierung innerhalb der Lebenswirklichkeit auf Grundlagen zu stellen, die von unserem Wahrnehmen, Fühlen, Wollen oder Entschliessen unabhängig sind und die uns verbindlich vorgegeben sind, scheint mir auch für Wilfried Härles ethisches Denken charakteristisch zu sein. In der Debatte über den ontologischen und moralischen Status des vorgeburtlichen Lebens sind es nach seiner Sicht die biologischen Eigenschaften von Embryonen, die diese zu Menschen und somit zu Trägern von Menschenwürde machen. ${ }^{11}$ In der Debatte über die Sterbehilfe sind es die kausalen Wirkungen einer ärztlichen Entscheidung, von denen nach seiner Sicht abhängt, ob es sich um aktive oder um passive Sterbehilfe handelt. ${ }^{12}$ Aus meiner Sicht wirft dies die Frage auf, ob man in Anbetracht unseres heutigen Wissens über Moral von einer solchen Objektivität ernstlich noch ausgehen kann. Es ist heute gesicherte Erkenntnis der empirischen Moralforschung in Psychologie und Neurobiologie, dass moralische Wertungen auf der emotionalen Bewertung von Handlungen und Situationen beruhen. ${ }^{13}$ Daher sind wir, wenn es um moralische Sachverhalte geht, immer schon subjektiv involviert. Was wir zum Beispiel mit den Sterbehilfeunterscheidungen verbinden, sind nicht Kausalabläufe, sondern emotional besetzte, normativ gehaltvolle situative ,Muster' oder Szenarien - ,Da wird ein Mensch getötet!', ,Mit diesem Therapieverzicht wird das Sterben eines Menschen zugelassen '-, die wir in konkreten Situationen und Handlungen wiedererkennen und die uns entsprechend auf diese eingestellt machen. $\mathrm{Ob}$ eine ärztliche Handlung aktive Sterbehilfe ist, das hängt daher davon ab, ob sie zu Recht unter diesem Muster wahrgenommen werden kann, und das erfordert einerseits eine Klärung dessen, was dieses Muster beinhaltet, und andererseits eine genaue Feststellung dessen, was der betreffende Arzt tatsächlich getan hat.

11 Vgl. Wilfried Härle, Menschenwürde - konkret und grundsätzlich, in: Ders., Menschsein in Beziehungen. Studien zur Rechtfertigungslehre und Anthropologie, Tübingen 2005, $381 \mathrm{ff}$.

12 Vgl. Wilfried Härle, Nein zur aktiven Sterbehilfe, in: zeitzeichen 3/2005, 811.

13 Vgl. Johannes Fischer, Grundlagen der Moral aus ethischer Perspektive und aus der Perspektive der empirischen Moralforschung, in: Ders./Stefan Gruden, Die Struktur der moralischen Orientierung. Interdisziplinäre Perspektiven, Berlin 2010, 19-48. 
Demgegenüber haben sich alle Versuche, die Sterbehilfeunterscheidungen an objektiven Eigenschaften von Handlungen festzumachen, als erfolglos erwiesen.

Dass es im Bereich der Moral keinen objektiven Standpunkt gibt, das zeigt sich vor allem daran, dass es keinen einzigen moralischen Begriff gibt, der sich rein begrifflich bestimmen lässt, das heißt ohne Rekurs auf die Anschauung oder Vorstellung, bei der wir immer schon emotional engagiert sind. Ein Beispiel ist der Begriff der Menschenwürde. Ersichtlich lässt sich der Gehalt dieses Begriffs nicht ohne den Rekurs auf die Anschauung beziehungsweise Vorstellung von Verletzungen bestimmen, die Menschen zugefügt werden können. Dieser Rekurs ist zum Beispiel wichtig im Blick auf Versuche, den Gehalt dieses Begriffs auf einen einzigen Aspekt zu reduzieren. So ist der Vorschlag gemacht worden, die Menschenwürde als das Recht aufzufassen, nicht erniedrigt, das heißt in seiner Selbstachtung verletzt zu werden. ${ }^{14}$ Ersichtlich ist diese Definition viel zu eng. Wenn im Bosnien-Krieg die serbische Soldateska mit Lastwagen über gefangene Muslime hinweg gefahren ist, dann betrachten wir dies zweifellos als eine Menschenwürdeverletzung. Doch soll diese tatsächlich darin bestanden haben, dass die Muslime durch diese Art der Tötung in ihrer Selbstachtung verletzt worden sind? Das mutet wie eine Verharmlosung des Geschehenen an. Die Muslime wurden schließlich auf brutale Weise umgebracht. Dasselbe lässt sich gegen die bei Kant zu findende Reduktion der Menschenwürde auf den Gedanken der Autonomie geltend machen. Soll die Menschenwürdeverletzung in diesem Beispiel sich tatsächlich darauf beschränken, dass die Autonomie der Muslime missachtet worden ist? Solche Beispiele verdeutlichen, wie problematisch ein begriffliches Denken auf dem Gebiet der Moral ist, das sich von der Anschauung und Vorstellung entfernt. Moralische Begriffe wie Grausamkeit, Erniedrigung oder Menschenwürde sind die sprachliche Artikulation erlebter beziehungsweise narrativ vergegenwärtigter Wirklichkeit, und sie können daher in ihrem Gehalt nur durch den Rückbezug auf diese Wirklichkeit expliziert werden, und dabei sind wir subjektiv involviert beziehungsweise emotional engagiert im Hinblick auf das, was sich uns solchermassen vor Augen stellt. Man mag sich an diesem Beispiel aus dem Bosnienkrieg die Bedeutung von Emotionen für die ethische Urteilskraft verdeutlichen. Es ist die emotionale Bewertung

14 Vgl. Peter Schaber, „Menschenwürde als das Recht, nicht erniedrigt zu werden“, in: Ralph Stoecker (Hg.), Menschenwürde - Annäherung an einen Begriff, Wien 2003, 119-131. 
des Geschehenen, die den Blick auf das Wesentliche lenkt und erkennen lässt, dass dessen Reduktion auf den Gedanken der Erniedrigung oder der Autonomie der Menschenwürdeverletzung, die hier geschehen ist, inadäquat ist. Hierin zeigt sich die kognitive Bedeutung von Emotionen. Sie generieren Erkenntnisse, die wir auf keine andere Weise gewinnen können. ${ }^{15}$

Ich habe zur Charakterisierung der Differenz zwischen Wilfried Härles Standpunkt und dem meinigen von der Unterscheidung „objektiv“-,,subjektiv“ Gebrauch gemacht. Vielleicht würde Wilfried Härle für die Charakterisierung seines Standpunkts den Begriff der Rationalität bevorzugen und dementsprechend an den meinigen die kritische Anfrage richten, inwiefern dieser dem Anspruch auf Rationalität genügt. Der Ausdruck ,Rationalität' ist mehrdeutig. Gemeint sein kann in dem hier in Rede stehenden Zusammenhang nur die argumentative Rationalität, im Unterschied zum Beispiel zur Zweckrationalität. Argumente sind Gründe, mit denen der Sprecher einem anderen zeigt, dass ein Urteil wahr oder dass es falsch ist. Wenn zutrifft, dass die Moral emotionale Grundlagen hat - was, wie gesagt, nach heutiger Erkenntnis als gesichert gelten muss -, dann kann die Wahrheit eines moralischen Urteils mit keinem Argument dieser Welt aufgewiesen werden. ${ }^{16}$ Vielmehr kann sie sich dann einem anderen nur selbst zeigen, indem er sich die betreffende Situation oder Handlung vor Augen führt beziehungsweise führen lässt und sie dabei emotional bewertet. Aus der Tatsache, dass moralische Wahrheit nicht argumentativ aufgewiesen werden kann, folgt jedoch nicht, dass es keine ethischen Argumente geben kann, im Gegenteil. Diesbezüglich muss zwischen Moral einerseits und Ethik als Moralreflexion andererseits unterschieden werden. So habe ich soeben gegen die Engführung des Gedankens der Menschenwürde auf den Gedanken der Erniedrigung oder der Autonomie argumentiert. Ich habe dies getan,

15 Vgl. hierzu Christoph Ammann, Emotionen - Seismographen der Bedeutung. Ihre Relevanz für eine christliche Ethik, Stuttgart 2007.

16 Diese Auffassung hat bereits vor einem Jahrhundert Harold Arthur PRICHARD mit scharfsinnigen Argumenten vertreten. Vgl. Ders., Beruht die Moralphilosophie auf einem Irrtum?, In: Günther Grewendorf/Georg Meggle (Hg.), Seminar: Sprache und Ethik. Zur Entwicklung der Metaethik, Frankfurt a.M. 1974, 6182. Prichard kommt zu dem Ergebnis, „dass wir nicht durch eine Argumentation (...) zur Erkenntnis einer Verpflichtung gelangen“ (a.a.O., 71). Vielmehr ist „,das Gefühl der Verpflichtung zu einer bestimmten Handlung oder die Richtigkeit dieser Handlung (...) absolut primär (d.h. von nichts anderem abgeleitet) beziehungsweise unmittelbar" (a.a.O., 69). 
indem ich jenes Beispiel aus dem Bosnienkrieg vor Augen gestellt habe, an dem sich dem Leser selbst zeigt, dass der Begriff der Menschenwürde weitaus umfassender ist, als solche Engführungen es nahe legen, und ich habe mit der Vergegenwärtigung dieses Beispiels und des sich darin Selbst-Zeigenden dem Leser gezeigt, dass es sich in dieser Weise verhält. Insofern habe ich mit diesem Beispiel argumentiert, und zwar ethisch argumentiert, insofern es um einen moralischen Begriff beziehungsweise Sachverhalt ging. Aber ich habe nicht rational im Sinne eines Verständnisses von ,Rationalität', das ohne Rekurs auf Anschauung und Vorstellung meint auskommen zu können, hergeleitet, dass die grausame Tötung von Menschen eine Menschenwürdeverletzung oder dass sie moralisch verwerflich ist. Die Vorstellung, man könne dies tun und moralische Urteile rational beziehungsweise argumentativ begründen, halte ich für eine Verirrung des Denkens, von der große Teile der heutigen Moralphilosophie erfasst sind. Sie hat zur Folge, dass sich die Ethik in begriffliche und gedankliche Abstraktionen verliert und blind wird für die Lebensphänomene. ${ }^{17}$ Man operiert dann mit einem Begriff wie ,Menschenwürde“ so, als ob es sich dabei um etwas objektiv, das heißt unabhängig von Anschauung und Vorstellung Gegebenes handeln würde, auf das man sich nach Belieben berufen kann. Die Folge ist begriffliche Verwilderung. Demgegenüber manifestiert sich moralische und ethische Sensibilität nach meinem Verständnis gerade im genauen Hinschauen auf die Situationen und Lebenslagen, in denen Menschen sich befinden. Das gilt insbesondere für den Bereich der Medizin, auf den vor allem sich die Kontroversen zwischen Wilfried Härle und mir bezogen haben.

Könnte es sein, dass es im Tiefsten die Sorge ist, dass alles subjektiv und beliebig wird, wenn der Anspruch auf eine rationale beziehungsweise objektive Rechtfertigung oder „Bewährung“ des Glaubens und der Moral aufgegeben wird, welche im Hintergrund von Wilfried Härles Auffassung von Theologie und Ethik steht und welche zu den Unterschieden im Grundsätzlichen unseres Denkens führt? Wenn es sich so verhält, dann verdient diese Sorge zweifellos großen Respekt. Gleich-

17 Vgl. dazu Johannes Fischer, Ethik als rationale Begründung der Moral? Über eine moralphilosophische Verirrung, http://www.ethik.uzh.ch/ise/publikationen/publikationen-1.html, letzter Zugriff am 10.8.2010. Es handelt sich um einen Text, den ich für eine interne Diskussion mit den Philosophinnen und Philosophen am Ethikzentrum der Universität Zürich geschrieben habe. Vgl. zu dieser Problematik auch: Johannes Fischer, Sittlichkeit und Rationalität. Zur Kritik der desengagierten Vernunft, Stuttgart 2010. 
wohl ist aus meiner Sicht zu fragen, ob wir uns der Einsicht entziehen können, dass die Lebenswirklichkeit nun einmal so verfasst ist, dass sie sich nur im persönlichen Involviertsein erschließt, und zwar sowohl im Bereich des Glaubens als auch im Bereich der Moral, und dass es keinen rationalen oder „objektiven“ Standpunkt ,dahinter“ gibt, den wir einnehmen könnten, um uns dessen zu vergewissern, was sich uns solchermaßen ,subjektiv“ erschlossen hat. Dies muss ja nicht bedeuten, dass wir uns nicht mit Gründen im Leben orientieren und intersubjektiv über diese Gründe verständigen können. Denn dieses Involviertsein vollzieht sich im Medium einer gemeinsamen Sprache, Kultur und religiösen Überlieferung. Das Beispiel der Menschenwürdeverletzung im Bosnienkrieg macht anschaulich, dass und wie solche Verständigung möglich ist.

Ein Letztes sei in diesem Zusammenhang noch angefügt. Ich habe den Unterschied zwischen Wilfried Härles Denken und dem meinigen bewusst nicht mit einem Begriffspaar gekennzeichnet, das sich hier ja ebenfalls nahe legen könnte, nämlich Realismus versus Antirealismus. Der Grund hierfür ist, dass ich mich ebenfalls als einen Realisten betrachte. Die Lebenswirklichkeit, wie ich sie soeben beschrieben habe, nämlich als etwas, das nur im persönlichen Involviertsein erschlossen ist, ist eine Realität. Daher trifft jenes Begriffspaar den Unterschied in unserem Denken nicht, und ich habe deshalb statt von ,Realismus' von ,Objektivismus' gesprochen im Sinne einer Auffassung, die mit dem Wort ,Realität‘ etwas verbindet, das unabhängig von unserem Wahrnehmen, Fühlen oder Erkennen gegeben ist. Dies ist die Vorstellung, die wir mit der natürlichen Welt verbinden, nämlich dass sie unabhängig von uns Menschen existiert. Der Mond ist rund, auch wenn es im ganzen Universum kein Wesen gäbe, das dies wahrnehmen könnte. Für mein Verständnis ist es eine Folge des Siegeszugs der modernen Naturwissenschaften, dass diese Auffassung von ,Realität` weithin bestimmend geworden ist auch für das Verständnis der menschlichen Lebenswirklichkeit. Hierdurch erst kommt es zu der irreführenden Alternative ,subjektiv-objektiv'. Das lässt sich zum Beispiel an der Wertphilosophie verfolgen, in der es eine Debatte darüber gibt, ob Werte „objektiv“ existieren oder ,subjektiv“ auf eine an sich wertneutrale Welt projiziert sind. Oder es lässt sich an dem Begriff ,natürlicher Eigenschaften“ in der Metaethik festmachen. Hiermit verbindet man Eigenschaften von Handlungen, Situationen oder Entitäten, die genauso objektiv wie die Eigenschaften des Mondes gegeben sind. Die Vorstellung ist dann, dass moralischer Wert supervenient mit diesen Eigenschaften gegeben ist, und 
das bedeutet, dass er genauso objektiv gegeben ist wie diese. ${ }^{18}$ Grausamkeit als ein thick moral concept ist dann mit den natürlichen Eigenschaften der grausamen Handlung gegeben, die moralische Qualität der aktiven Sterbehilfe ist mit der kausalen Eigenschaft der betreffenden Handlungen, den Tod eines Menschen zu bewirken, gegeben, die Menschenwürde ist mit Eigenschaften wie der Vernunftfähigkeit oder der Handlungsfähigkeit ${ }^{19}$ des Menschen gegeben, oder im Falle von Embryonen mit deren biologischen Eigenschaften. Ich möchte Wilfried Härle nichts unterstellen und es daher in Gestalt einer Frage formulieren, die sich mir aufgrund seiner medizin- und bioethischen Texte aufdrängt: Könnte es sein, dass er aus der genannten Sorge bezüglich subjektiver Willkür und Beliebigkeit heraus diese Art von „objektivistischem“ Realismus vertritt? Könnte es sein, dass er es aufgrund dieser Sorge unbedingt vermeiden möchte, dass es letztlich von unserem ,subjektiven“ Bewerten abhängt, was moralisch gut oder was moralisch richtig ist, und dass er deshalb das Gute und Richtige - paradigmatisch etwa in Gestalt der Menschenwürde - als etwas „objektiv“ Gegebenes festhalten möchte?

Wie dem auch sei - aus meiner Sicht liegt das Fragwürdige dieser Art des Denkens darin, dass es aus einer notwendigen eine hinreichende Bedingung macht. Zum Vergleich: Lichtwellen müssen eine bestimmte Wellenlänge haben, damit wir die Farbe, rot' wahrnehmen. Aber Röte ist nicht bereits mit dieser Wellenlänge des Lichts gegeben, also unabhängig von unserer Wahrnehmung. Ebenso kann man sagen, dass ein Verhalten bestimmte natürliche Eigenschaften aufweisen muss, um als grausam erlebt und wahrgenommen werden zu können. Aber Grausamkeit ist nicht mit diesen Eigenschaften gegeben, das heißt unabhängig davon, wie wir Verhalten erleben und wahrnehmen und dabei emotional bewerten. Die emotionale Bewertung richtet sich auf die erlebte beziehungsweise

18 Man kann diese Auffassung, welche in der Metaethik verbreitet ist, mit Fug und Recht als ,Naturalismus' bezeichnen. Allerdings muss man dann diese Art von Naturalismus, welche moralischen Wert auf natürliche Eigenschaften zurückführt, über denen er superveniert, vom metaethischen Naturalismus im engeren Sinne unterscheiden, welcher eine Variante des moralischen Realismus darstellt und der sich auf die Bedeutung moralischer Ausdrücke bezieht. ,Gut' wird dann z. B. als entweder bedeutungs- oder als sachidentisch mit dem Ausdruck, fördert das Wohl von Personen" interpretiert. Insbesondere in der bioethischen Debatte ist ein Naturalismus im ersten, weitgefassten Sinne verbreitet, wie man sich an den SKIP-Argumenten verdeutlichen kann.

19 Vgl. Alan Gewirth, Human Rights: Essays of Justification and Applications, Chicago 1982. 
narrativ thematisierte Realität, im Unterschied zur objektivierten, deskriptiv thematisierten Realität, auf die sich die Rede von ,natürlichen Eigenschaften' bezieht. Das bedeutet, dass auch moralischer Wert, insofern er auf emotionaler Bewertung beruht, nicht unabhängig von unserem Erleben gegeben ist. Daher kann er nicht mit etwas gegeben sein, das hiervon ersichtlich unabhängig ist, nämlich mit natürlichen Eigenschaften. Hieraus folgt jedoch nicht, dass moralischer Wert „bloss subjektiv" ist. Wir können uns intersubjektiv darüber verständigen, ob eine Handlung grausam ist. Wir können dies, weil das Wort ,grausam“ die sprachliche Artikulation eines (Wahrnehmungs-)Musters ist, das wir gemeinsam über die Sprache internalisiert haben. Es bezieht sich, wie gesagt, auf die erlebte Realität, und wir können es in vielen einzelnen Handlungen wiedererkennen. Dass eine Handlung grausam ist, das hat seine hinreichende Bedingung darin, dass sie dieses Muster aktualisiert, das seinerseits gewisse natürliche Eigenschaften im Sinne einer notwendigen Bedingung voraussetzt dafür, dass Handlungen unter diesem Muster wahrgenommen werden können. Das Urteil „Diese Handlung ist grausam“ erhebt also wie das Urteil „Dieser Teppich ist rot“ durchaus einen Objektivitätsanspruch in dem Sinne, dass wir mit ihm nicht bloß unsere subjektive Sicht der Dinge kommunizieren. Aber dieser Anspruch bezieht sich nicht auf eine unabhängig von unserem Wahrnehmen und Erkennen gegebene Realität, sondern auf die für unser gemeinsames Wahrnehmen und Erkennen gegebene Realität, die durch eine gemeinsame Sprache und Kultur verbürgt ist. So, wie es im Fall der Röte das uns von Natur gemeinsame Sehvermögen ist, das uns dieselbe Farbe sehen lässt, so gibt es auch ein Sehvermögen, das uns aufgrund unserer kulturellen Prägung gemeinsam ist. Insofern halte ich die Sorge für unbegründet, dass alles subjektiv und beliebig wird, wenn man die Lebenswirklichkeit in der oben beschriebenen Weise versteht, wonach sie nur dem persönlichen Engagement erschlossen ist. Ich bin demgegenüber der Ansicht, dass das Modell des „objektivistischen“ Realismus den Blick auf die Lebenswirklichkeit gründlich verstellt. In theologischer Hinsicht bedeutet dies, dass die entscheidende Frage nicht ist, wie wir das christliche Wirklichkeitsverständnis oder wie wir die Moral „objektiv“realistisch rechtfertigen oder bewähren können, sondern vielmehr, wodurch wir uns in unserem Blick auf die Dinge bestimmen lassen. Diese Frage fuihrt in den Bereich der Pneumatologie. Der sachliche Zusammenhang von Ethik und Pneumatologie ist trotz der diesbezüglichen neutestamentlichen Befunde in großen Teilen der evangelischen Ethik bis vor Kurzem kein Thema gewesen, vermutlich aufgrund der Meinung, 
dass wissenschaftliche Ethik es mit rationalen Begründungen zu tun hat und nicht mit derlei obskuren Dingen wie dem Heiligen Geist. Heute ist zwar die "Spiritualität" in vieler Munde, und dieses Thema hat auch in Bereichsethiken wie die Pflegeethik und selbst in die Wirtschaftsethik Einzug gehalten, aber wenn es sich hierbei um mehr als nur eine Modeerscheinung handeln soll, dann bedarf es einer gründlichen fundamentalethischen Klärung dieses Zusammenhangs.

Es kann vorkommen, dass man sich am Morgen einer Sache noch gewiss war. Am Mittag meldeten sich erste Zweifel, und am Abend ist von der Gewissheit nichts mehr übrig, und die Suche beginnt von Neuem. Wie sicher können wir uns dessen sein, was uns zum jetzigen Zeitpunkt evident zu sein scheint? Über dieser Frage kann man erschrecken, da wir ja auch eine Verantwortung tragen für die Ideen, die wir in die Welt setzen. Letztlich können wir immer nur den vorläufigen Stand der uns möglichen Einsicht formulieren. In diesem Sinne verstehe ich die kritische Weggefährtenschaft, die mich mit Wilfried Härle verbindet. Wir beide haben in unseren theologischen und ethischen Kontroversen doch nichts anderes tun können, als - jeder auf seine Weise - den vorläufigen Stand der uns möglichen Einsicht zu artikulieren, immer unter dem Vorbehalt des möglichen Irrtums. Je älter man wird, umso skeptischer wird man gegenüber dem Anspruch auf endgültige Wahrheiten. Viel zu lange hat die Theologie - jedenfalls zu großen Teilen - den Gestus der Gewissheit gepflegt, so als könne man von der Sache, die sie zu verantworten hat, nur in dieser Weise sprechen. Keine andere Wissenschaft hat sich das leisten können und zu dergleichen je verstiegen. Das war zwar für die Bildung theologischer Schulen förderlich, die ihre je eigenen Gewisheiten kultivierten und die Gewissheiten der Konkurrenz bekämpften, nicht aber für das kritische Denken, das in aller Regel mit dem Zweifel beginnt. In der evangelischen Ethik hat dies teilweise zu einem Klima geführt, in dem ein unvoreingenommener, sachlicher und vor allem fairer und respektvoller Umgang in hochkontroversen ethischen Fragen unmöglich gewesen ist, wie er für eine Wissenschaftskultur selbstverständlich sein sollte. ${ }^{20}$ Ich weiß nicht, ob ich in dieser Ein-

20 Ich denke hier insbesondere an die Debatten über den ontologischen und moralischen Status des vorgeburtlichen menschlichen Lebens, wie sie durch die 
schätzung mit Wilfried Härle völlig einig gehe, aber ich hoffe es. Auch wenn wir uns trotz grosser Anstrengungen im Grundsätzlichen unseres theologischen und ethischen Denkens nicht haben verständigen können, und auch wenn uns in unseren materialen ethischen Überzeugungen vieles trennt, so achte und schätze ich die Ernsthaftigkeit und Leidenschaft, mit der es ihm um die Sache der Theologie zu tun ist. Ich wünsche ihm zu seinem Siebzigsten alles Gute!

Stammzellforschung ausgelöst worden sind. Wer dabei war, der mag sich diesbezüglich an den Europäischen Theologenkongress 2002 in Zürich erinnern. Ein Grundproblem scheint mir darin zu liegen, dass sich solche Debatten in der Regel auf materialethische Fragen konzentrieren, ohne dass die fundamentaltheologischen und fundamentalethischen Positionen, die dabei im Hintergrund stehen und an denen sich die Differenzen zum wesentlichen Teil entzünden, zum Thema gemacht und einer eingehenden Reflexion unterzogen werden. So prallen bei jedem neuen materialethischen Thema die eingefahrenen Fundamentalüberzeugungen unverändert aufeinander. 